\title{
Evaluation of Point of Need Diagnostic Tests for Use in California Influenza Outbreaks
}

\author{
Ashlynn Daughton* and Alina Deshpande
}

A1, Los Alamos National Lab, Los Alamos, NM, USA

\section{Objective}

Evaluate utility of point of need diagnostic tests in relationship to current standard influenza detection methods.

\section{Introduction}

Each year several thousands contract the seasonal flu, and it is estimated that these viruses are responsible for the deaths of over six thousand individuals [1]. Further, when a new strain is detected (e.g. 2009), the result can be substantially more dramatic [2]. Because of the potential threats flu viruses pose, the United States, like many developed countries, has a very well established flu surveillance system consisting of 10 components collecting laboratory data, mortality data, hospitalization data and sentinel outpatient care data [3]. Currently, this surveillance system is estimated to lag behind the actual seasonal outbreak by one to two weeks. As new data streams come online, it is important to understand what added benefit they bring to the flu surveillance system complex. For data streams to be effective, they should provide data in a more timely fashion or provide additional data that current surveillance systems cannot provide.

Two types of multiplexed diagnostic tools designed to test syndromically relevant pathogens and wirelessly upload data for rapid integration and interpretation were evaluated to see how they fit into the influenza surveillance scheme in California.

\section{Methods}

Data Acquisition

Percent influenza like illness data was obtained for California from the CDC website as well as local California public health websites from 2014 to 2015. Point of need (PON) data was obtained by the Biosurveillance Ecosystem and included data about tests run, and results of tests.

Outbreak identification

Flu data from California was split into discrete outbreaks based on the CDC's current flu outbreak definition [3].

Data Stream Analysis

For each outbreak, the date that point of need diagnostic data is available was compared to the CDC's flu surveillance data. Further analyses will examine outbreak trends between PON data and the CDC's data to determine if PON data is capable of detecting outbreaks earlier than standard methods.

\section{Results}

Figure 1 shows one comparison of point of need (PON) data to the current public health standard in San Diego. The green line shows the weekly percent ILI in San Diego during the 2014 to 2015 flu season. The orange line show the total number of PON records (or number of PON tests conducted) during the same outbreak, and the purple line shows the number of those records that detected the flu (i.e. were positive). Similar analyses will be conducted for the upcoming 2015-2016 flu season, and will be conducted for other locations in California, both retrospectively and prospectively.

\section{Conclusions}

This data will be used to evaluate point of need data streams in influenza outbreaks in California. We will be able to determine if they provide additional useful data that can be used to identify outbreaks earlier, or if they do not add beneficial data to the influenza surveillance system.

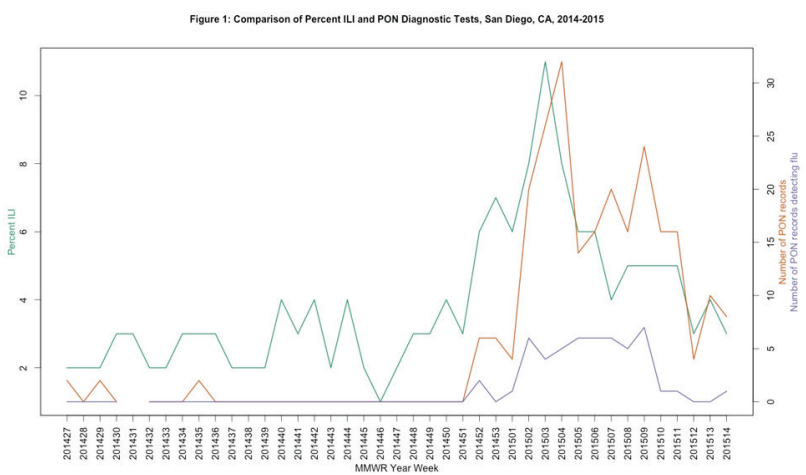

Figure 1: Shows a comparison of influenza like illness data in San Diego from 2014 to 2015 (green), corresponding point of need records/ tests run (orange), and the number of records that were positive (purple)

\section{Keywords}

Point of need diagnostic; Influenza; Surveillance; California

\section{Acknowledgments}

This project is funded by the Defense Threat Reduction Agency (DTRA). The data was provided as a part of the Biosurveillance Ecosystem (BSVE).

\section{References}

1. Centers for Disease Control and Prevention (CDC). Estimates of deaths associated with seasonal influenza —- United States, 1976-2007. MMWR Morb. Mortal. Wkly. Rep. 59, 1057-1062 (2010).

2. Fineberg, H. V. Pandemic Preparedness and Response - Lessons from the H1N1 Influenza of 2009. New England Journal of Medicine 370, 1335-1342 (2014).

3. Overview of Influenza Surveillance in the United States. (2007). at $<$ http://www.cdc.gov/flu/weekly/pdf/flu-surveillance-overview.pdf $>$

\section{*Ashlynn Daughton}

E-mail: adaughton@lanl.gov 